\title{
A Note on $\lambda_{2}$ and $\lambda_{n}$ of a Graph
}

\author{
MiRJAnA LAZiĆ
}

\begin{abstract}
Using the eigenvalues and eigenvectors of a graph $G$, it was established the upper bound for the second eigenvalue $\lambda_{2}$ and the least eigenvalue $\lambda_{n}$ [1]. In this work using only the eigenvalues of $G$ we obtain the upper bound for $\lambda_{2}$ and $\lambda_{n}$.
\end{abstract}

Let $G$ be a graph of order $n$ and let $A$ be its ordinary adjacency matrix. The spectrum of $G$ is the set of its eigenvalues $\lambda_{1} \geq \lambda_{2} \geq \ldots \geq \lambda_{n}$. We say that $\lambda_{i}$ is the $i$-th eigenvalue of $G(i=1,2, \ldots, n)$. In particular, $\lambda_{2}$ is called the second eigenvalue while $\lambda_{n}$ is called the least eigenvalue. Using the eigenvalues and eigenvectors of $G$ it was obtained the upper bound for $\lambda_{2}$ and $\lambda_{n}$ (see [1], p. 222). In this paper we obtain the upper bound for $\lambda_{2}$ and $\lambda_{n}$ using only the eigenvalues of $G$.

Theorem 1 ([3]). Let $G$ be a graph of order $n$, and let $\left\{\lambda_{i}\right\}$ and $\left\{\bar{\lambda}_{i}\right\}$ be the corresponding eigenvalues of $G$ and its complement $\bar{G}$, respectively. Then:

$$
\begin{gathered}
\lambda_{i}+\bar{\lambda}_{n+1-i}+1 \geq 0 \quad(i=1,2, \ldots, n), \\
\lambda_{i+1}+\bar{\lambda}_{n+1-i}+1 \leq 0 \quad(i=1,2, \ldots, n-1) .
\end{gathered}
$$

Theorem 2. If $G$ is a graph with $n$ vertices then $\lambda_{2} \leq \frac{n-2}{2}$.

Proof. Assume, on the contrary, that there exists a graph $G$ of order $n$ with $\lambda_{2}>\frac{n-2}{2}$. Let $S=\frac{1}{n-2} \sum_{i=3}^{n}\left|\lambda_{i}\right|$. Then we can see that

$$
(n-2) S^{2} \leq \sum_{i=3}^{n}\left|\lambda_{i}\right|^{2} .
$$

Since

$$
\sum_{\lambda_{i}<0}\left|\lambda_{i}\right|=\sum_{\lambda_{i}>0}\left|\lambda_{i}\right|
$$

2000 Mathematics Subject Classification. Primary: 51M16.

Key words and phrases. geometric inequalities, fundamental inequalities of triangle, Gerretsen's inequalities. 
and

we have

$$
\lambda_{1} \geq \lambda_{2}>\frac{n-2}{2}
$$

$$
S \geq \frac{1}{n-2} \sum_{\lambda_{i}<0}\left|\lambda_{i}\right|=\frac{1}{n-2} \sum_{\lambda_{i}>0}\left|\lambda_{i}\right| \geq \frac{1}{n-2}\left(\lambda_{1}+\lambda_{2}\right)>1 .
$$

From relations (3) and (4) we have

$$
\sum_{i=3}^{n}\left|\lambda_{i}\right|^{2} \geq(n-2) \cdot S^{2}>n-2
$$

Let $m$ and $\bar{m}$ be the numbers of edges of the graphs $G$ and $\bar{G}$, respectively. Then from (5) and using relations (1) and (2), we find that:

$$
\begin{aligned}
n^{2}-n & =2 m+2 \bar{m}=\sum_{i=1}^{n} \lambda_{i}^{2}+\sum_{i=1}^{n} \bar{\lambda}_{i}^{2} \geq \lambda_{1}^{2}+\lambda_{2}^{2}+\sum_{i=3}^{n} \lambda_{i}^{2}+\bar{\lambda}_{1}^{2}+\bar{\lambda}_{n}^{2}> \\
& >2\left(\frac{n-2}{2}\right)^{2}+(n-2)+2\left(\frac{n}{2}\right)^{2}=n^{2}-n,
\end{aligned}
$$

which is a contradiction.

Corollary 1. If $\lambda_{1} \in\left(\frac{n-2}{2}, n-1\right]$ then $\lambda_{1}$ is the simple eigenvalue.

Further, let $G$ be non-regular graph of order $n$. We know that $\lambda_{1}(G)=d(G)+$ $\Delta(G)$, where $d(G)$ denotes the mean value of the vertex degrees of $G$ and $\Delta(G)>$ 0 . In view of this,

$$
\lambda_{1}(G)+\lambda_{1}(\bar{G})=n-1+\Delta(G)+\Delta(\bar{G}) .
$$

The proof of the next result is based on a property of the so-called canonical graphs [2].

We say that two vertices $x, y \in V(G)$ are equivalent in $G$ and write $x \sim y$ if $x$ is non-adjacent to $y$, and $x$ and $y$ have exactly the same neighbors in $G$. Relation $\sim$ is an equivalence relation on the vertex set $V(G)$. The corresponding quotient graph is denoted by $\widetilde{G}$, and is called the canonical graph of $G$.

We say that $G$ is canonical if $|G|=|\widetilde{G}|$, that is if $G$ has no two equivalent vertices. Let $\widetilde{G}$ be the canonical graph of $G,|\widetilde{G}|=k$, and $N_{1}, N_{2}, \ldots, N_{k}$ be the corresponding sets of equivalent vertices in $G$. Then we denote $G=\widetilde{G}\left(N_{1}, N_{2}, \ldots, N_{k}\right)$, or simply $G=\widetilde{G}\left(n_{1}, n_{2}, \ldots, n_{k}\right)$, where $\left|N_{i}\right|=n_{i}(i=1,2, \ldots, k)$.

In the case that $\left|N_{i}\right|=m$ for $\left.i=1,2, \ldots, k\right)$, the corresponding graph $\widetilde{G}(m, m, \ldots, m)$ is denoted by $G_{m k}$. With this notation in [2] was proved the following result:

Proposition 1. Let $\widetilde{G}$ be a canonical graph of order $k$, and let $\left\{\lambda_{i}\right\}$ and $\left\{\bar{\lambda}_{i}\right\}$ be the corresponding eigenvalues of $\widetilde{G}$ and its complement $\widetilde{G}$, respectively. Then 
$\left(1^{\circ}\right) H_{G_{m k}}(t)=m H_{\widetilde{G}}(m t)$;

$\left(2^{\circ}\right) \sigma\left(G_{m k}\right)=\left\{m \lambda_{i} \mid i=1,2, \ldots, k\right\} \bigcup\{\underbrace{0,0, \ldots, 0}_{n-k}\}$;

$\left(3^{\circ}\right) \sigma\left(\bar{G}_{m k}\right)=\left\{m \bar{\lambda}_{i}+m-1 \mid i=1,2, \ldots, k\right\} \bigcup\{\underbrace{-1,-1, \ldots,-1}_{n-k}\}$,

where $H_{G}(t)$ is the generating function of the numbers of walks in the graph $G$.

Lemma 1. Let $G$ be non-regular graph of order $n$. Then for every $M>0$ there exists a graph $G^{*} \supseteq G$ of order $n^{*}=m \cdot n$ such that

$$
\lambda_{1}\left(G^{*}\right)=d\left(G^{*}\right)+\Delta\left(G^{*}\right) \geq d\left(G^{*}\right)+M,
$$

and

$$
\lambda_{1}\left(G^{*}\right)+\lambda_{1}\left(\bar{G}^{*}\right) \geq n^{*}-1+M .
$$

Proof. Let $\left\{\lambda_{i} \mid i=1,2, \ldots, n\right\}$ be the eigenvalues of $G$. Then $\left\{m \lambda_{i} \mid i=\right.$ $1,2, \ldots, n\} \bigcup\{\underbrace{0,0, \cdots, 0}_{n^{*}-n}\}$ are the eigenvalues of $G^{*}$. We now obtain the proof using the fact that $\lambda_{1}\left(G^{*}\right)=m \cdot \lambda_{1}(G)$ and $d\left(G^{*}\right)=m \cdot d(G)$.

Theorem 3. If $G$ is a non-regular graph with $n$ vertices then $\left|\lambda_{n}\right|<\frac{n}{2}$.

Proof. We can suppose, on the contrary, that there exists a non-regular graph $G$ of order $n$ whit $\left|\lambda_{n}\right| \geq \frac{n}{2}$. Let $m$ be the least integer such that $\Delta\left(G^{*}\right) \geq 2$. Then, $\left|\lambda_{n}\left(G^{*}\right)\right| \geq \frac{n^{*}}{2}$, where $n^{*}$ is the order of $G^{*}$. For $k \in N$ we consider $G_{k}^{*}=\bigcup_{i=1}^{k} G^{*}$. Then $\left|G_{k}^{*}\right|=k \cdot n^{*}=k \cdot m \cdot n$, and its eigenvalues are $m \cdot \lambda_{1} \geq m \cdot \lambda_{2} \geq \cdots \geq m \cdot \lambda_{n}$ of multiplicity $k$, while 0 is the eigenvalue of $G^{*}$ of the multiplicity $k \cdot n^{*}-k \cdot n$.

Now, we have

(6) $n^{* 2} \cdot k^{2}-n^{*} \cdot k=\sum_{i=1}^{n^{*} k} \lambda_{i}^{* 2}+\sum_{i=1}^{n^{*} \cdot k} \bar{\lambda}_{i}^{* 2}=k \lambda_{1}^{* 2}+\cdots+k \lambda_{n}^{* 2}+k \bar{\lambda}_{1}^{* 2}+\cdots+k \bar{\lambda}_{n}^{* 2}$.

Using relations (1), (2) and (6) we have

$$
n^{* 2} \cdot k^{2}-n^{*} \cdot k \geq k \lambda_{1}^{* 2}+k\left(\frac{n^{*}}{2}\right)^{2}+\bar{\lambda}_{1}^{* 2}+(k-1)\left(\frac{n^{*}}{2}-1\right)^{2}+(k-1)\left(\lambda_{1}+1\right)^{2} .
$$

Using (7) by an easy calculation we find that

$$
n^{* 2} \cdot k^{2}-n^{*} \cdot k \geq k \cdot \frac{n^{* 2}}{2}-\frac{n^{* 2}}{4}+2(k-1)+f\left(\lambda_{1}, \bar{\lambda}_{1}\right),
$$

where $f\left(\lambda_{1}, \bar{\lambda}_{1}\right) \equiv(2 k-1) \lambda_{1}^{* 2}+\bar{\lambda}_{1}^{* 2}$.

Next, we obtain that

$$
\min f\left(\lambda_{1}, \bar{\lambda}_{1}\right)=n^{* 2} \cdot k^{2}-\frac{k \cdot n^{* 2}}{2}+\frac{(2 k-1)\left(\Delta_{*}-1\right)^{2}}{2 k}+n^{*}(2 k-1)\left(\Delta_{*}-1\right) .
$$


Since $\lambda_{1}^{*}+\bar{\lambda}_{1}^{*}=n^{*} k-1+\Delta_{*}$, where $\Delta_{*}=\Delta\left(G_{k}^{*}\right)+\Delta\left(\bar{G}_{k}^{*}\right) \geq \Delta\left(G_{k}^{*}\right) \geq 2$, we get

$$
n^{* 2} \cdot k^{2}-n^{*} \cdot k \geq n^{* 2} k^{2}-\frac{n^{* 2}}{4}+(2 k-1)+n^{*} \cdot(2 k-1),
$$

a contradiction.

Corollary 2. For every regular graph $G,\left|\lambda_{n}\right| \leq \frac{n}{2}$.

Proof. We can assume, on the contrary case, that there exists a regular graph $G$ of order $n$, with $\left|\lambda_{n}\right|>\frac{n}{2}$. Let $\left|\lambda_{n}\right|=\frac{n}{2}+\varepsilon \quad(\varepsilon>0)$. Then there exists a graph $G^{*}$ of order $n^{*}=m \cdot n$ so that

$$
\left|\lambda_{n}\left(G^{*}\right)\right|=m \cdot\left|\lambda_{n}(G)\right|>\frac{n^{*}}{2}+1
$$

Let $G_{*}=G^{*} \cup K_{1}$, where $K_{1}$ is the graph with one isolated vertex. Since $G_{*}$ is non-regular and according to theorem 3 ,

$$
\left|\lambda_{n}\left(G_{*}\right)\right|<\frac{n\left(G_{*}\right)}{2}=\frac{n^{*}+1}{2},
$$

we get a contradiction to relation (9).

\section{REFERENCES}

[1] D.Cvetković, M.Doob and H.Sachs, Spectra of Graphs-Theory and Applications, revised and enlarged edition, J.A.Barth Verlag, Heidelberg-Leipzig, 1995.

[2] M.Lepović, On canonical graphs and eigenvectors of graphs, Collection of scientific papers of the Faculty of Science Kragujevac, 21(1999) 75-85.

[3] M.Lepović, On eigenvalues and main eigenvalues of a graph, Math. Moravica, Vol. 4(2000), $51-58$

Department of Mathematics

AND INFORMATICS

Faculty of Sciences

P.O. Box 60

34000 Kragujevac

SERBia AND Montenegro

E-mail address: mmmvl@kg.ac.yu 